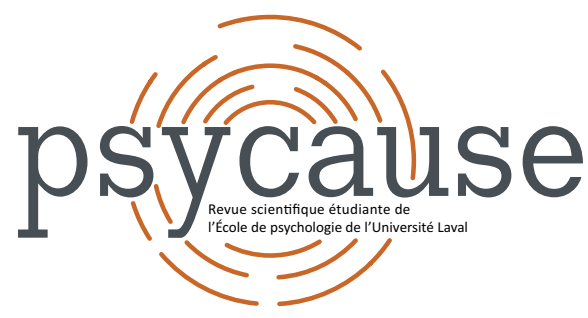

\section{PSYCAUSE}

Revue scientifique étudiante de l'École de psychologie de l'Université Laval

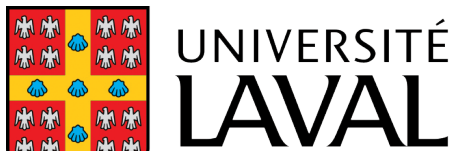

Faculté des sciences sociales École de psychologie

\title{
IMPACT DE LA COMORBIDITÉ DANS LE TRAITEMENT DES JOUEURS PATHOLOGIQUES
}

\author{
Noémie DANSEREAU TOUCHETTE ${ }^{1 \star}$ \\ 'École de psychologie, Université Laval, Québec, QC, Canada \\ *noemie.dansereau-touchette.1@ulaval.ca
}

\section{Pour citer l'article}

Dansereau Touchette, N. (2021). Impact de la comorbidité dans le traitement des joueurs pathologiques. Psycause: Revue scientifique étudiante de l'École de psychologie de l'Université Laval, 11(1), $15-23$

\section{Droits d'auteur}

(C) 2021 Dansereau Touchette. Cet article est distribué en libre accès selon les termes d'une licence Creative Commons Attribution 4.0 International (de type CC-BY 4.0) qui permet I'utilisation du contenu des articles publiés de façon libre, tant que chaque auteur ou autrice du document original à la publication de l'article soit cité(e) et référencé(e) de façon appropriée. 


\title{
IMPACT DE LA COMORBIDITÉ DANS LE TRAITEMENT DES JOUEURS PATHOLOGIQUES
}

\author{
Noémie DANSEREAU TOUCHETTE ${ }^{*}$ \\ 'École de psychologie, Université Laval, Québec, QC, Canada \\ *noemie.dansereau-touchette.1@ulaval.ca
}

\section{Résumé}

\begin{abstract}
Les jeux de hasard et d'argent ne cessent de se développer à travers le monde et l'accroissement de leur visibilité permet un portrait plus juste des variables impliquées dans la problématique du trouble lié au jeu d'argent. Les données probantes quant à la compréhension de ce trouble se développent rapidement et le jeu d'argent pathologique se présente majoritairement en cooccurrence avec d'autres troubles de santé mentale. Les résultats de recherches ont démontré que jusqu'à $96 \%$ des personnes ayant reçu un diagnostic de jeu d'argent pathologique ont satisfait aux critères d'au moins un autre trouble psychiatrique au cours de leur vie (Bischof et al., 2013; Kessler et al., 2008; Steel et Blaszczynski, 1998). Par ailleurs, les troubles d'utilisation de substances (TUS) ont un taux de prévalence particulièrement élevé chez les joueurs pathologiques (Cowlishaw et al., 2014; Fernández, 2002) et cette concomitance peut avoir un impact important sur la problématique de même que sur l'issue d'un traitement. De plus, les joueurs pathologiques ne représentent pas un groupe homogène et plusieurs caractéristiques les distinguent entre eux. Ce sont ces caractéristiques qui demandent à être mieux comprises. Ainsi, l'objectif de cet article est d'identifier les différences étiologiques chez les joueurs en lien avec l'impact de la comorbidité, particulièrement le TUS associé au jeu d'argent pathologique (JAP) et leurs influences sur les programmes de traitement.
\end{abstract}

\section{Mots-clés : joueur pathologique, comorbidité, traitement, recension, jeux de hasard et d'argent}

\section{Abstract}

Gambling continues to gain in popularity around the world and the increased visibility provides a more accurate picture of the variables involved in the problem of gambling disorder. Evidence to understand this disorder is growing rapidly, and pathological gambling is unique in that it occurs predominantly in co-occurrence with other mental health disorders. Research have shown that up to $96 \%$ of people diagnosed with pathological gambling met criteria for at least one other psychiatric disorder in their lifetime (Bischof et al., 2013; Kessler et al., 2008; Steel and Blaszczynski, 1998). In addition, substance use disorders (SUD) have a particularly high prevalence rate among pathological gamblers (Cowlishaw et al., 2014; Fernández, 2002) and this concomitance can have a strong impact on the disorder itself as well as on the outcome of treatment. Pathological gamblers do not represent an homogeneous group and several characteristics distinguish them from each other. These characteristics need to be better understood. Thus, the objective of this article is to identify the etiological differences in gamblers in relation to the impact of the comorbidity, particularly the SUD associated with pathological gambling (JAP) and their influence on treatment programs.

\section{Keywords : pathological gambler, comorbidity, treatment, review, gambling}

L'offre de jeux de hasard et d'argent ne cesse de se développer à travers le monde et l'accessibilité grandissante augmente le risque de développer des comportements addictifs (Chevalier et Allard, 2001; Gijsen et al., 2013). La disponibilité des données probantes quant à la compréhension du jeu d'argent pathologique (JAP) a également suivi cette accélération. Aujourd'hui, la littérature scientifique soutien que le JAP a la particularité de se retrouver majoritairement en cooccurrence avec d'autres troubles de santé mentale tels que le trouble d'utilisation de substances (TUS), les troubles de l'humeur, les troubles anxieux et les troubles de la personnalité (Kessler et al., 2008; Lorains et al., 2011; Pelletier, 2007; Petry et al., 2005; Rush et al., 2008; Sacco et al., 2008). La recherche à ce sujet démontre que jusqu'à $96 \%$ des personnes ayant reçu un diagnostic de trouble lié au JAP ont satisfait aux critères d'au moins un autre trouble psychiatrique au cours de leur vie (Bischof et al., 2013; Kessler et al., 2008; Steel et Blaszczynski, 1998). De ce fait, la forte association entre le TUS et les joueurs pathologiques est soutenue dans la littérature scientifique (Cowlishaw et al., 2014; Fernández, 2002; Kessler et al., 2008; Lorains et al., 2011; Petry, 2005). Cette concomitance peut avoir un impact important dans le développement de la problématique de même que dans le maintien des gains associés au traitement ou à la complétion de celui-ci. En effet, lorsque les deux troubles se retrouvent simultanément chez une même personne, la littérature démontre une augmentation de la sévérité clinique, ce qui provoque une capacité de traitement plus complexe ainsi qu'une augmentation de la prévalence des rechutes (Ferland et al., 2018; 
Hodgins et el-Guebaly, 2010; Wieczorek et Dąbrowska, 2020). Les comorbidités asociées au JAP agissent en amont et en aval de la problématique faisant fluctuer son intensité. Conséquemment, nombreuses sont les variables qui influencent la sévérité du JAP et ces mécanismes doivent être mieux compris afin d'en faciliter la modélisation. Or, les traitements actuellement disponibles font abstraction de la forte comorbidité entre le JAP et d'autres troubles (Pelletier et al., 2008). Cette conception ayant une validité interne unique et délimitée, ne permet pas une validité externe optimale et par conséquent, propose une vision restreinte ne reflétant pas la réalité propre au joueur pathologique. Cette optique peut également entrainer des effets délétères sur celui-ci. En effet, les traitements offerts peuvent eux-mêmes expliquer les taux élevés d'abandon au traitement (Ball et al., 2006). D'une part, il existe un enjeu à traiter les joueurs pathologiques ayant un double diagnostic dans un court laps de temps et dans un délai normalisé. Étant donné la sévérité clinique importante pour cette catégorie de joueur, le traitement peut être moins efficace. D'autre part, l'absence de lignes directrices pour les joueurs pathologiques atteints de troubles comorbides demeure préoccupante. Dans ce contexte, le but de cet article consiste à faire le point sur les connaissances actuelles concernant l'impact de la comorbidité dans le traitement pour joueurs pathologiques. Plus précisément, cette recension a pour objectifs de mettre en lumière les différentes variantes étiologiques du joueur pathologique en concordance avec l'impact du TUS sur le traitement du JAP.

\section{L'hétérogénéité des joueurs pathologiques}

\section{Typologie des joueurs pathologiques}

Bien que les psychopathologies et les traits de personnalités diffèrent entre les joueurs pathologiques, ceux-ci ont principalement été étudiés de façon homogène (Milosevic et Ledgerwood, 2010). Pour mieux conceptualiser le joueur pathologique, il est nécessaire d'illustrer les sous-groupes afin de comprendre les implications étiologiques, le traitement et la gravité du trouble.

Un des modèles les plus acceptés et utilisés dans la compréhension de l'hétérogénéité des joueurs adultes est celui proposé par Blaszczynski et Nower (2002; Milosevic et Ledgerwood, 2010). Ce modèle, validé par Milosevic et Ledgerwood (2010), propose des variables développementales, neurobiologiques et cognitives ce qui en fait un modèle explicatif complet. II existe trois typologies distinctes de joueurs selon ces auteurs. D'abord, le joueur au comportement conditionné (CC) est caractérisé par une absence de pathologie prémorbide, ayant des problèmes de jeu davantage transitoires et présenterait moins d'abus de substances et moins d'impulsivité. Ensuite, le joueur émotionnellement vulnérable (EV) présente des comorbidités préexistantes telles que l'anxiété et la dépression, a de faibles capacités d'adaptation, révèle davantage un historique de milieu familial défavorable et a vécu des évènements de vie éprouvants. Finalement, le joueur antisocial impulsif (Al), quant à lui, se définit par une vulnérabilité biologique amenant davantage d'impulsivité, ainsi que par des troubles de la personnalité antisociale, des troubles attentionnels et des comportements sévèrement inadaptés (Blaszczynski et Nower, 2002). Or, chaque sous-groupe présente des caractéristiques qui doivent être prises en compte dans le traitement.

Chez le joueur de type CC, le développement de la problématique serait associé à l'exposition répétée au jeu et son maintien aux contingences comportementales (Blaszczynski et Nower, 2002). Ainsi, les problèmes de jeu sont moins sévères et sont moins susceptibles de subir l'interaction d'une psychopathologie sous-jacente. Les joueurs de type $\mathrm{CC}$ oscillent entre le jeu à risque et le JAP, mais en raison d'un bon fonctionnement prémorbide, ils sont plus enclins à vivre du succès dans le traitement (Ledgerwood et Petry, 2010).

Les joueurs de type EV ont des précurseurs similaires au type CC. Cependant, ils utilisent le jeu comme moyen afin de moduler un affect douloureux. Ainsi, ils sont plus résistants au changement de comportement et les interventions doivent inclure à la fois un traitement ciblant les vulnérabilités problématiques reliées au jeu et les vulnérabilités reliées à l'humeur et aux affects (Blaszczynski et Nower, 2002).

Finalement, les joueurs de type Al ont également une vulnérabilité émotionnelle, mais présentent davantage une dysrégulation émotionnelle reliée de près aux troubles de la personnalité limite et antisociale. Or, contrairement aux autres sous-types, les joueurs de type Al sont caractérisés par des niveaux élevés d'impulsivité, un dysfonctionnement neurologique qui, en combinaison avec des psychopathologies sous-jacentes, provoquent une combinaison étiologique unique qui explique l'apparition et le maintien du jeu (Blaszczynski et Nower, 2002). Ainsi, le traitement pour ce type de joueur inclut non seulement le jeu, mais également un éventail de traitements pour d'autres dysfonctionnements, tels les troubles de la personnalité et l'impulsivité (Blaszczynski et Nower, 2002).

La sévérité des problèmes de jeu est supérieure pour les joueurs de type EV et Al (Ledgerwood et Petry, 2010). Bien que les joueurs de type $C C$ répondent mieux au traitement en raison de la moins grande sévérité de la cooccurrence de difficultés psychosociales, Ledgerwood et Petry (2010) ont démontré que lorsqu'ils sont en traitement, les trois sous- 
groupes s'améliorent à un rythme similaire. Par contre, en raison de leur sévérité initialement plus élevée, les joueurs de type EV et Al montrent une gravité élevée du JAP à la fin du traitement et pendant la période du suivi. Comme le mentionnent Ledgerwood et Petry (2006), chaque sousgroupe présente des caractéristiques qui leur sont propres et peut nécessiter une approche de traitement différente. Par ailleurs, considérant que les joueurs de type EV et Al ont des difficultés psychosociales plus sévères (Ledgerwood et Petry, 2010) et que le TUS est une comorbidité largement associée à ces types de joueurs (Blaszczynski et Nower, 2002), il est important de comprendre l'interaction de ces deux troubles et leurs mécanismes sous-jacents afin de raffiner les traitements qui leur sont destinés.

\section{Le trouble d'utilisation de substances et le jeu d'argent pathologique: similitudes, différences et prévalence}

Il existe de nombreuses similitudes entre le trouble d'utilisation de substances et le jeu d'argent pathologique, particulièrement au niveau des critères diagnostiques dont cinq sont communs entre le TUS et le JAP. Les concordances sont également présentes au niveau neurobiologique par des déficits cognitifs dans les fonctions exécutives et d'inhibition du contrôle tels que l'impulsivité et la compulsion (Bosc et al., 2012; Leeman et Potenza, 2012; Nicholson et al., 2019; Rash et al., 2016). Ainsi, en raison des similitudes étiologiques et théoriques entre ces deux troubles, la prévalence du JAP serait plus élevée parmi les individus présentant un TUS que dans le reste de la population (Arsenault et al., 2001).

Malgré leurs similitudes, les deux troubles doivent être compris séparément, car le traitement du JAP cible uniquement les spécificités propres au joueur pathologique. La caractéristique fondamentale qui démarque le JAP du TUS sont les erreurs cognitives propres au trouble et qui amène une évaluation erronée de la réalité. Toneatto et ses collaborateurs (1997) ont identifié 13 distorsions cognitives qui influencent le maintien des comportements compulsifs de jeu. La plus fréquemment citée de par son importance (Barrault, 2012; Bosc et al., 2012; Lucas, et al., 2011; Toneatto et al., 1997), l'illusion de contrôle, se définit comme étant des comportements ou pensées superstitieuses qui amènent les gens à croire qu'ils influencent l'issue du jeu, niant ainsi la part importante de chance et de hasard. Cela amène les joueurs à attribuer leurs succès à des évènements internes (Barrault, 2012). Les profils sociodémographiques et la sévérité clinique diffèrent lorsque les deux troubles sont en concomitance (Ferland et al., 2018). Or, il existe des enjeux cliniques et thérapeutiques lorsque les deux troubles se présentent ensemble.

Dans une étude de Petry et ses collaborateurs (2005), la prévalence pour le trouble d'utilisation de l'alcool (abus et dépendance) était de $73 \%$ chez les JP, tandis que l'étude de Kessler et ses collaborateurs (2008) présentait plutôt une prévalence de $46 \%$ pour l'abus de drogue et alcool et de $32 \%$ pour la dépendance. Dans une étude combinant une revue systématique et une méta analyse, Lorains et ses collaborateurs (2011) ont trouvé un taux de prévalence de $58 \%$ pour le TUS chez les joueurs pathologiques. Or, bien que les taux varient d'une étude à l'autre, en moyenne, un joueur pathologique sur deux présente un TUS (Bronnec, 2010).

\section{Influence du trouble d'utilisation de substances et jeu d'argent pathologique sur le traitement}

\section{Sévérité clinique}

Un des plus grands impacts lorsque le JAP et le TUS se présentent simultanément demeure l'augmentation de la sévérité clinique. Cette enchevêtrement des deux troubles amène un éventail de difficultés plus importantes dans le processus de traitement (Ferland, et al., 2018; Lister et al., 2015). En effet, en comparant deux groupes mutuellement exclusifs, soit le groupe jeu sans TUS et le groupe jeu avec TUS, Ferland et al. (2018) montrent que les groupes ayant les deux troubles simultanément présentent des besoins en intervention plus importants sur plusieurs plans (p. ex., dégradation des relations interpersonnelles, présence de difficultés psychologiques, professionnelles, financières et judiciaires) et que la concomitance mènerait à une aggravation des difficultés vécues.

Les personnes atteintes de JAP ont également une prévalence plus élevée de symptômes d'anxiété et de dépression qui aggravent les difficultés de traitement (Parhami et al., 2014; Petry et al., 2005). La présence accrue de symptômes d'anxiété et de dépression augmente donc la sévérité clinique et, par conséquent, doit être prise en compte dans le traitement. En outre, interroger les comportements de jeu et intervenir dans les premiers stades du JAP peut empêcher la progression vers des formes plus graves du JAP et prévenir l'apparition d'une comorbidité future (Parhami et al., 2014).

Par ailleurs, certains individus atteints du JAP et du TUS peuvent également omettre de dire I'une ou l'autre des dépendances (Wieczorek et Dąbrowska, 2020). Ainsi, lors d'un traitement, l'intervention demeurera partielle puisqu'elle ne ciblera qu'une partie de la problématique. $\mathrm{Ne}$ pas traiter simultanément le JAP et le TUS peut agraver I'un des deux troubles (Cowlishaw et al., 2014; Wieczorek et Dąbrowska, 2020). Ainsi, ces difficultés illustrent bien l'impact de la cooccurrence du jeu et du TUS lors du traitement.

L'association du JAP et du TUS, lorsque présent simultanément chez une même personne, provoque une interaction 
biologique et comportementale engendrant une augmentation de la sévérité clinique (Barnes et al., 2005; Vitaro et al., 2007). Vitaro et ses collaborateurs (2007) montrent un lien fort entre la consommation de substances psychoactive, les comportements délinquants et les problèmes reliés au jeu. Notamment, la force des relations entre ces trois comportements reste sable de 16 à 23 ans et le lien entre la pratique des JAP et les comportements délinquants augmente. Différents facteurs de risque à l'enfance pourraient expliquer la relation entre ces catégories. Or, il est possible de faire le lien avec le joueur de type Al de Blaszczynski et Nower (2002), sachant que ce type de joueur présente une sévérité clinique accrue et, par conséquent, une capacité de traitement plus difficile et complexe. De plus, plusieurs études ont mis en évidence le rôle de l'impulsivité, particulièrement au niveau de la sévérité des habitudes de jeu, de la consommation de substances psychoactives et du développement de ceux-ci (Gendron, 2012). Nombreuses sont les études ayant démontré une forte relation entre le JAP et un haut niveau d'impulsivité (Blanco et al., 2009; Gendron, 2012; Rash et al., 2016; Vitaro et al., 2007). De ce fait, les analyses de Dussault et ses collaborateurs (2011) ont révélé un lien prédictif positif entre l'impulsivité à l'âge de 14 ans, les symptômes dépressifs et les problèmes de jeu à l'âge de 17 ans. À son tour, les problèmes de jeu à l'âge de 17 ans prédisent une augmentation des symptômes dépressifs de 17 à 23 ans et des symptômes dépressifs à l'âge 17 ans prédisent une augmentation des problèmes de jeu de 17 à 23 ans. Également, Blanco et ses collaborateurs (2009) ont montré une corrélation positive entre le niveau d'impulsivité et la sévérité des symptômes du JAP. Conséquemment, l'impulsivité serait une variable médiatrice, car elle accentue la prise de risques, mène à une sévérité du JAP plus élevée et augmente la consommation de substances psychoactives (van Hamel et al., 2007). Ainsi, le lien entre la consommation et le JAP est médié par une impulsivité biologique plus élevée et peut mener à une pratique plus sévère des jeux de hasard et d'argent. Le traitement pour joueurs pathologiques est associé à une diminution des symptômes du JAP mais a également amoindrit l'impulsivité (Blanco et al., 2009). Par conséquent, lors du traitement, il semblerait que des changements dans l'impulsivité pourraient jouer un rôle médiateur sur la gravité des symptômes du JAP (Blanco et al., 2009). Ainsi, il est important d'évaluer l'interaction entre l'impulsivité, la consommation de substances psychoactives et la sévérité des habitudes de jeu en raison de leur influence sur l'issue du traitement.

\section{Efficacité des traitements incluant des problématiques de dépendances variées}

Or, qu'en est-il des personnes atteintes de jeu pathologique sans comorbidité avec le TUS qui se retrouvent en traitement dans les groupes incluant des problématiques de dépendances variées? Tout d'abord, il est important de souligner l'importance de la cohésion du groupe lors d'une thérapie. En effet, plus la cohésion de groupe est forte, meilleure est l'amélioration de la condition des participants. (Burlingame et al., 2011). Cependant, l'hétérogénéité des problématiques de dépendance affaiblirait la force du sentiment de cohésion (Légaré et al., 2019). Le discours des participants fait également mention d'aspects négatifs sur leur expérience lors d'un traitement incluant différentes dépendances (Chevalier et al., 2003; Légaré et al., 2019). Ils mentionnent que la thérapie aurait été davantage pertinente et efficace si celle-ci avait exclusivement été centrée sur leur dépendance personnelle (Légaré, 2018).

Il est également important de mentionner que l'utilisation des groupes incluant des problématiques de dépendances variées apparaît inopportun en raison de l'absence d'intervention sur les composantes spécifiques du traitement cognitif comportemental du JAP (Légaré et al., 2019). En effet, ces composantes ne sont pas présentées de manière systématique par les thérapeutes, malgré qu'elles soient identifiées comme étant importantes dans le processus de rétablissement des joueurs (Légaré et al., 2019). Ainsi, le traitement des groupes incluant des problématiques de dépendances variées semble peu approprié pour le joueur pathologique compte tenu de ses caractéristiques spécifiques qui ne sont pas prises en compte dans le traitement.

\section{L'abandon du traitement chez les joueurs pathologiques}

Dans une revue systématique comprenant 12 études, Melville et ses collaborateurs (2007) ont montré que l'abandon au sein d'études de traitement psychologique du JAP varie entre 14 et $50 \%$. De plus, Slutske (2006) rapporte que seulement $7 \%$ à $12 \%$ des personnes ayant reçu un diagnostic de JAP avaient demandé un traitement formel ou assisté à des réunions de Gamblers Anonymes. Certains auteurs tels que Hodgins et el-Guebaly (2000) ou Slutske (2006) croient que ce faible taux serait attribuable au fait que $33 \%$ à $36 \%$ des joueurs pathologiques se rétablissent sans traitement. Cependant, les joueurs pathologiques ayant une sévérité clinique faible ou modérée préfèrent ne pas suivre de traitement (Toneatto et al., 2008). Selon le modèle proposé par Blaszczynski et Nower (2002), il semble que les joueurs s'étant rétablis sans traitement ressemblent le plus étroitement aux joueurs $\mathrm{CC}$ qui ont généralement moins de psychopathologies prémorbides (Toneatto et al., 2008).

L'évaluation d'un traitement du JAP d'approche cognitive comportementale implanté au Québec a révélé un taux d'abandon de 5,5\% des joueurs en services internes et $65 \%$ des joueurs en services externes (Allard et Papineau, 2006). 
Cela signifie que plus de la moitié des joueurs en traitement à l'externe abandonne le traitement avant sa fin. Or, il faut faire la distinction entre les motifs d'abandon et les variables possiblement prédictives de l'abandon. Le premier réfère aux motifs propres à l'usager (p. ex., manque de temps, la difficulté de transport, etc.), au traitement (p. ex., programme trop répétitif, programme de groupe uniquement, etc.), à l'intervenant (p. ex., manque de rigueur, de formation, d'expérience, etc.) et ne dégage pas de portrait statistiquement significatif (Allard et Papineau, 2006; Demers-Labonté, 2017; Giroux et al., 2015; Melville et al., 2007). Cependant, les motifs d'abandon nous informent que la comorbidité influence la trajectoire personnelle du joueur pathologique en modulant ses actions et perceptions. Quant aux variables possiblement prédictrice de l'abandon, les facteurs psychologiques et psychosociaux (p. ex., la dépression, les troubles de personnalité, les abus d'alcool et de drogue, le stade de changement, les comorbidités, l'anxiété et la détresse psychologique) jouent un rôle prépondérant quant à l'issue du traitement (Bergeron et al., 1997; Juneau, 2006; Merkouris et al., 2016). Les recherches sur les variables possiblement prédictives de l'abandon permettent de mieux comprendre l'enjeu des comorbidités et des sous-groupes, tel que décrit par Blaszczynski et Nower (2002) dans la compréhension de l'abandon, particulièrement élevé chez les joueurs pathologiques. En effet, plusieurs auteurs rapportent que présenter une comorbidité est prédictif de l'issue du traitement (Echeburúa et al., 2001; Hodgins et el-Guebaly, 2010; Merkouris et al., 2016). Plus particulièrement, les troubles de personnalité ainsi que le TUS sont deux comorbidités fortement corrélées à l'abandon et à la difficulté de maintenir l'abstinence (Hodgins et el-Guebaly, 2010; Giroux et al., 2015; Merkouris et al., 2016; Milton et al., 2002; Pelletier, 2007). Le jeu, combiné à la consommation d'alcool, entrấnerait un renforcement négatif significatif, lequel est particulièrement résistant au traitement et associé à un abandon prématuré (Milton et al., 2002).De plus, ne pas considérer les comorbidités sous-jacentes au JAP dans le traitement constitue une variable prédictive de l'issue du traitement (Légaré et al., 2019; Merkouris et al., 2016). Par ailleurs, tel que rapporté par Leblond et ses collaborateurs (2003), l'impulsivité, une variable importante dans le TUS et le JAP, est significative et prédit l'abandon au traitement. Ceci appuie également la pertinence des sous-groupes de Blaszcznski et Nower (2002), car les joueurs Al et EV montrent un niveau d'impulsivité supérieur et une moins grande réponse au traitement (Ledgerwood et Petry, 2010). Enfin, l'interaction entre le JAP et le TUS doit être mieux comprise afin de développer et suggérer des traitements efficaces.

Plusieurs recommandations ont déjà été portées à l'attention des instances quant à l'amélioration du traitement pour les joueurs pathologiques. De ce fait, au Québec, le Guide des bonnes pratiques de l'Association des centres de réadaptation en dépendance du Québec (2008) propose, entre autres, que les interventions auprès des joueurs pathologiques s'inscrivent dans un modèle biopsychosocial, allant de la pharmacothérapie aux approches psychosociales. Reconnaître la comorbidité dans l'intervention clinique permet de maximiser la réponse au traitement, améliore la satisfaction du patient et diminue son attrition (Dowling et al., 2015; Maniaci et al., 2017).

\section{Limites}

Il faut toutefois demeurer prudent quant aux résultats des études présentées. Le manque d'uniformité dans les études ne permet pas une généralisation adéquate aux populations de joueurs pathologiques. En effet, il est important de mentionner que la notion d'abandon du traitement ne fait pas consensus auprès des chercheurs. Selon certains auteurs, l'abandon se produit avant le début du traitement (Robson et al., 2002), pour d'autres il survient pendant le traitement (Echeburúa et al., 2001). Ainsi, les taux de variabilité rapportés d'une étude à l'autre dépendent de la définition proposée par les auteurs. Le manque de cohérence dans la définition de l'abandon rend difficile la comparaison des résultats entre les études ainsi que leur réplication. Par conséquent, ces études ne permettent pas d'offrir un tableau juste et clair de la situation (Demers-Labonté, 2017; Giroux et al., 2015; Melville et al., 2007).

Également, le taux de prévalence lorsque les TUS et le JAP sont en comorbidités diffère d'une étude à l'autre. Cela s'explique, entre autres, par le fait que les études n'utilisent pas les mêmes outils pour mesurer la dépendance. En effet, certaines études incluent le trouble d'abus de substances (Lorains et al., 2011), d'autres utilisent une mesure pour l'abus et la dépendance (Petry et al., 2005), ou encore, certaines mesurent uniquement l'abus aux drogues et à l'alcool (Kessler et al., 2008). Puisque les outils mesurent des réalités différentes, cela influence l'interprétation des résultats et réduit sa généralisation. Or, il devient impératif d'uniformiser les définitions tant pour l'abandon que pour les mesures reliées au TUS afin de mieux comparer les études entres elles et ainsi améliorer la compréhension des variables impliquées dans le JAP. Ce n'est qu'avec un portrait précis que l'efficience des traitements deviendra optimale.

\section{Conclusion}

La littérature scientifique montre l'hétérogénéité de la problématique et ses conséquences lors du traitement. Les différences étiologiques du JAP ne semblent pas s'harmoniser avec les programmes de traitement standardisés et, par conséquent, peuvent être un facteur explicatif du fort taux 
d'abandon. De plus, le taux élevé de comorbidité associé au JAP, particulièrement le TUS, augmente la sévérité et dans de graves circonstances, le traitement peut même avoir un effet iatrogène (Wieczorek et Dąbrowska, 2020). Également, le traitement incluant des dépendances variées fait état d'une non-concordance entre les spécificités propres aux joueurs et les interventions reliées au traitement. Or, les difficultés reliées à la concomitance de ces deux troubles ne permettent pas le traitement optimal. Considérant les taux élevés d'abandon lors du traitement (Melville et al., 2007; Allard et Papineau, 2006) et la faible recherche d'aide (Slutske, 2006), la plupart des études se sont concentrées à identifier les variables prédictives de l'abandon ou les obstacles à la recherche d'aide. Cependant, il serait intéressant de comprendre comment ces joueurs ont surmonté les obstacles à la demande d'aide et quels sont les facteurs associés au maintien du traitement. Une meilleure compréhension des facteurs de protection favorisant le maintien en traitement permettrait d'optimiser les stratégies de traitements et d'en bonifier la conceptualisation.

\section{Références}

Allard, D., \& Papineau, É. (2006). Évaluation du programme expérimental sur le jeu / Rapport synthèse. Institut national de santé publique du Québec.

Arsenault, L., Ladouceur, R., \& Vitaro, F. (2001). Jeu de hasard et consommation de substances psychotropes: prévalence, coexistence et conséquences. Canadian Psychology, 43(3), 173-184. https://doi.org/10.1037/h0086890

Association des centres de réadaptation en dépendance du Québec. (2008). Les services en jeu pathologique dans les centres de réadaptation en dépendance; guide des bonnes pratiques et offre de services de base. Association des centres de réadaptation en dépendance du Québec.

Ball, S., Carroll, K., Canning-Ball, M., \& Rounsaville, B. (2006). Reasons for dropout from drug abuse treatment: Symptoms, personality, and motivation. Addictive Behaviors, 31(2), 320-330. https://doi.org/10.1016/j.addbeh.2005.05.013

Barnes, G., Welte, J., Hoffman, J., \& Dintcheff, B. (2005). Shared predictors of youthful gambling, substance use, and delinquency. Psychology of Addictive Behaviors, 19(2), 165174. https://doi.org/10.1037/0893-164X.19.2.165

Barrault, S. (2012). Étude des distorsions cognitives, des troubles anxiodépressifs et de la personnalité chez des joueurs pathologiques en ligne et hors ligne. Université Paris Descartes.
Bergeron, J., Landry, M., Brochu, S., \& Cournoyer, L.-G. (1997). Les déterminants de la persévérance des clients dans les traitements de réadaptation pour l'alcoolisme et la toxicomanie: une approche multidimentionnelle. Rapport d'une recherche subventionnée par le Conseil québécois de la recherche sociale

Bischof, A., Meyer, C., Bischof, G., Kastirke, N., John, U., \& Rumpf, H.-J. (2013). Comorbid Axis I-disorders among subjects with pathological, problem, or at-risk gambling recruited from the general population in Germany: Results of the PAGE study. Psychiatry Research, 210(3), 1065-1070. https://doi.org/10.1016/j.psychres.2013.07.026

Blanco, C., Potenza, M., Kim, S., Ibáñez, A., Zaninelli, R., SaizRuiz, J., \& Grant, J. (2009). A pilot study of impulsivity and compulsivity in pathological gambling. Psychiatry Research, 167(1-2), 161-168. https://doi.org/10.1016/j. psychres.2008.04.023

Blaszczynski, A., \& Nower, L. (2002). A pathways model of problem and pathological gambling. Addiction, 97(5), 487499. https://doi.org/10.1046/j.1360-0443.2002.00015.x

Bosc, E., Fatséas, M., Alexandre, J.-M., \& Auriacombe, M. (2012). Similitudes et différences entre le jeu pathologique et la dépendance aux substances: qu'en est-il ? L'Encéphale, 38(5), 433-439. https://doi.org/10.1016/j. encep.2012.02.004

Bronnec, M. R.-L. (2010). Jeu et addiction. Annales Médico-Psychologiques, revue psychiatrique, 168(7), pp. 509-512. https://doi.org/10.1016/j.amp.2010.06.004

Burlingame, G., McClendon., D., \& Alonso, J. (2011). Cohesion in Group Therapy. Psychotherapy, 48(1), 34-42. https://doi. org/10.1037/a0022063

Chevalier, S., \& Allard, D. (2001). Jeu pathologique et joueurs problématiques; Le jeu à Montréal. Direction de la santé publique, Régie régionale de la santé et des services sociaux de Montréal-Centre, Montréal .

Chevalier, S., Geoffrion, C., Audet, C., Papineau, É., \& Kimpton, M.-A. (2003). Évaluation du programme expérimental sur le jeu pathologique. Rapport 8 - Le point des usagers. Institut national de santé publique., Montréal.

Costes, J.-M., Pousset, M., Eroukmanoff, V., Le Nezet, O., Richard, J., Guignard, R., Beck, F., \& Arwidson, P. (2011). Les niveaux et pratiques des jeux de hasard et d'argent en 2010. Tendances, 77. https://www.ofdt.fr/publications/ collections/periodiques/lettre-tendances/niveaux-et-pratiques-jeux-de-hasard-et-argent-en-2010-tendances-77septembre-2011/

Cowlishaw, S., Merkouris, S., Chapman, A., \& Radermacher, H. (2014). Pathological and problem gambling in substance use treatment: A systematic review and meta-analysis. Journal of Substance Abuse Treatment, 46(2), 98-105. https://doi.org/10.1016/j.jsat.2013.08.019 
Cox.J., B., Yu, N., Afifi, T., \& Ladouceur, R. (2005). A National Survey of Gambling Problems in Canada. The Canadian Journal of Psychiatry, 50(4), 213-217. https://doi. org/10.1177/070674370505000404

Demers-Labonté, B. (2017). Synthèse des connaissances sur l'abandon d'un traitement par les joueurs de jeux de hasard et d'argent. [Mémoire doctoral, Université Laval]. https://corpus.ulaval.ca/jspui/bitstr eam/20.500.11794/27881/1/33360.pdf

Dowling N., Cowlishaw, S., Jackson, A., Merkouris, S., Francis, K., \& Christensen, D. (2015). Prevalence of psychiatric co-morbidity in treatment-seeking problem gamblers: A systematic review and meta-analysis. Australian \& New Zealand Journal of Psychiatry, 49(6), 519-539. http://doi. org/10.1177/0004867415575774

Echeburúa, E., Fernandez-Montalvo, J., \& Baez, C. (2001). Predictors of therapeutic failure in slot-machine pathological gamblers following behavioural treatment. Behavioural and Cognitive Psychotherapy, 29, 379-383. http://doi. org/10.1017/S1352465801003113

Ferland, F., Blanchette-Martin, N., Savard, A.-C., Vézina, É., Légaré, A.-A., Champagne, A., Baillargeon-Lemieux, H., Giroux, I., \& Garceau, P. (2018). Les joueurs pathologiques en traitement sont-ils un groupe homogène? Différences selon la concomitance d'un trouble d'utilisation d'une substance. Journal of Gambling Issues, 39, 124-146. http:// dx.doi.org/10.4309/jgi.2018.39.11

Fernández, D. G.-S. (2002). La comorbidité chez les joueurs pathologiques en traitement. [Mémoire de maîtrise, Université de Montréal]. https://papyrus.bib.umontreal.ca/ xmlui/handle/1866/8897

Gendron, A. (2012). Influence de la sévérité de la consommation de substances psychoactives, de la détresse psychologique et de l'impulsivité sur la sévérité des habitudes de jeux de jeux de hasard et d'argent à l'adolescence. [Thèse de doctorat, Université du Québec à Trois-Rivières] http:// depot-e.uqtr.ca/id/eprint/6168/1/030403993.pdf

Gijsen, P., Jammaer, R., Mourad, H., \& Pitchot, W. (2013). Le jeu pathologique: Evolution conceptuelle, caractéristiques cliniques et perspectives psychothérapeutiques. Revue Médicale de Liège, 68(5-6), 359-364. Récupéré sur https:// orbi.uliege.be/bitstream/2268/196458/1/Jeu\%20pathologique.pdf

Giroux, I., Bouchard, S., \& Sévigny, S. (2015). Synthèse des connaissances sur l'entrée, la persistance et l'abandon des joueurs pathologiques en traitement. Québec, Université Laval.

Hodgins, D., \& el-Guebaly, N. (2010). The Influence of Substance Dependence and Mood Disorders on Outcome from Pathological Gambling: Five-Year Follow-Up. Journal of Gambling Studies, 26, 117-127. http://doi.org/10.1007/ s10899-009-9137-9
Hodgins D., \& el-Guebaly, N. (2000). Natural and Treatment-assisted Recovery From Gambling Problems: A Comparison of Resolved and Active Gamblers. Addiction, 95, 777-789. https://doi.org/10.1046/j.1360-0443.2000.95577713.x

Juneau, S. (2006). Motivation et persévérance au traitement; le cas des joueurs pathologiques du Saguenay-Lac-St-Jean. [Mémoire de maîtrise, Université du Québec en Outaouais.]

Kairouz, S., Paradis, C., Nadeau, L., Hamel, D., \& Robillard, C. (2015). Patterns and trends in gambling participation in the Quebec population between 2009 and 2012. Revue Canadienne de Santé Publique, 106(3), 115-120. http:// doi.org/10.17269/CJPH.106.4854

Kessler, R., Hwang, I., LaBrie, R., Petukhova, M., Sampson, N., Winters, K., et Shaffer, H. (2008). The prevalence and correlates of DSM-IV Pathological Gambling in the National Comorbidity Survey Replication. Psychological Medicine, 38(9), 1351-1360. http://doi.org/10.1017/ S0033291708002900

Leblond, J., Ladouceur, R., \& Blaszczynski, A. (2003). Which pathological gamblers will complete treatment? British Journal of Clinical Psychology, 42(2), 205-209. https://doi. org/10.1348/014466503321903607

Ledgerwood, D., \& Petry, N. (2006). Psychological experience of gambling and subtypes of pathological gamblers. Psychiatry Research, 144(1), 17-27. https://doi.org/10.1016/j. psychres.2005.08.017

Ledgerwood, D., \& Petry, N. (2010). Subtyping pathological gamblers based on impulsivity, depression and anxiety. Psychology of Addictive Behaviors, 24(4),680-688. https:// psycnet.apa.org/doi/10.1037/a0019906

Leeman, R., \& Potenza, M. (2012). Similarities and Differences between Pathological Gambling and Substance Use Disorders: A Focus on Impulsivity and Compulsivity. Psychopharmacology, 219(2), 469-490. http://doi.org/10.1007/ s00213-011-2550-7

Légaré, A.-A. (2018). Traitements de groupes incluant des joueurs et des toxicomanes: Retombées du traitement et exploration des facteurs thérapeutiques. [Thèse de doctorat, Université Laval]. https://corpus.ulaval.ca/jspui/bitstr eam/20.500.11794/29951/1/34154.pdf

Légaré, A.-A., Giroux, I., \& Ferland, F. (2019). Traitement en groupe incluant des joueurs et des toxicomanes: atteinte des objectifs thérapeutiques et retombées. Journal of Gambling Issues, 41, 124-146. http://dx.doi.org/10.4309/ jgi.2019.41.7

Lister, J., Milosevic, A., \& Ledgerwood, D. (2015). Personality traits of problem gamblers with and without alcohol dependence. Addictive Behaviors, 47, 48-54. https://doi. org/10.1016/j.addbeh.2015.02.021 
Lorains, F., Cowlishaw, S., \& Thomas, S. (2011). Prevalence of comorbid disorders in problem and pathological gambling: systematic review and meta-analysis of population survey. Addiction, 106(3), 490-498. https://doi.org/10.1111/ j.1360-0443.2010.03300.x

Lucas, C., Lucia, R., Legauffre, C., Nichols, E., Morvannou, A., \& Adès, J. (2011). Distorsions cognitives dans le jeu récréatif et problématique. Etat de la question. Alcoologie et addictologie, 33(4), 325-331.

Maniaci G., La Cascia, C., Picone, F., Lipari, A., Cannizzaro, C., \& La Barbera, D. (2017). Predictors of early dropout in treatment for gambling disorder: The role of personality disorders and clinical syndromes. Psychiatry Research, 257, 540-545. https://doi.org/10.1016/j.psychres.2017.08.003

Melville, K., Casey, L., \& Kavanagh, D. (2007). Psychological treatment dropout among pathological gamblers. Clinical Psychology Review, 27(8), 944-958. https://doi. org/10.1016/j.cpr.2007.02.004

Merkouris, S., Thomas, S., Browning, C., \& Dowling, N. (2016). Predictors of outcomes of psychological treatments for disordered gambling: A systematic review. Clinical Psychology Review, 48, 7-13. http://dx.doi.org/10.1016/j. cpr.2016.06.004

Milosevic, A., \& Ledgerwood, D. (2010). The subtyping of pathological gambling: A comprehensive review. Clinical Psychology Review, 30(8), 988-998. https://doi.org/10.1016/j. cpr.2010.06.013

Milton, S., Rocco, C., Hunt, C., \& Prosser, E. (2002). The Effect of Compliance-Improving Interventions on the Cognitive-Behavioural Treatment of Pathological Gambling. Journal of Gambling Studies, 18(2), 207-229. http://doi. org/10.1023/a:1015580800028

Nicholson, R., Mackenzie, C., Aff, T., Keough, M., \& Sareen, J. (2019). An Examination of Comorbid Psychiatric Disorders in Disordered Gamblers Versus Other Substance Related Disorders. Journal of Gambling Studies, 35(3), 829-847. https://doi.org/10.1007/s10899-019-09839-y

Parhami, I., Mojtabai, R., Rosenthal, R., Afifi, T., \& Fong, T. (2014). Gambling and the Onset of Comorbid Mental Disorders: A Longitudinal Study Evaluating Severity and Specific Symptoms. Journal of Psychiatric Practice, 20(3), 207-219. http:// doi.org/10.1097/01.pra.0000450320.98988.7c

Pelletier, O. (2007). Trouble de la personnalité et jeu pathologique: comorbidité et prédicteurs d'abandon du traitement. [Thèse de doctorat, Université Laval]. https://corpus. ulaval.ca/jspui/handle/20.500.11794/20351

Pelletier, O., Ladouceur, R., \& Rhéaume, J. (2008). Personality Disorders and Pathological Gambling: Comorbidity and Treatment Dropout Predictors. International Gambling Studies, 8(3), 299-313. https://doi. org/10.1080/14459790802405913
Petry, N. (2005). Pathological gambling: Etiology, comorbidity, and treatment. American Psychological Association.

Petry, N., Stinson, F., \& Grant, B. (2005). Comorbidity of DSM-IV Pathological Gambling and Other Psychiatric Disorders: Results From the National Epidemiologic Survey on Alcohol and Related Conditions. The Journal of clinical psychiatry, 66(5), 564-574. https://doi.org/10.4088/JCP.v66n0504

Rash, C., Weinstock, J., \& Van Patten, R. (2016). A review of gambling disorder and substance use disorders. Substance Abuse and Rehabilitation, 7, 3-13. https://doi.org/10.2147/ SAR.S83460

Robson E., Edwards, J., Smith, G., \& Colman, I. (2002). Gambling decisions: An early intervention program for problem gamblers. Journal of Gambling Studies, 18(3), 235-255. http:// doi.org/10.1023/A:1016888705033

Rush, B., Bassani, D., Urbanoski, K., \& Castel, S. (2008). Influence of co-occurring mental and substance use disorders on the prevalence of problem gambling in Canada. Addiction, 103(11), 1847-1856. https://doi.org/10.1111/j.13600443.2008.02338.x

Sacco, P., Cunningham-Williams, R., Ostmann, E., \& Spitznagel, E. (2008). The association between gambling pathology and personality disorders. Journal of Psychiatric Research, 42(13), 1122-1130. https://doi.org/10.1016/j. jpsychires.2007.11.007

Slutske, W. (2006). Natural Recovery and Treatment-Seeking in Pathological Gambling: Results of Two U.S. National Surveys. The American Journal of Psychiatry, 163(2), 297-302. https://doi.org/10.1176/appi.ajp.163.2.297

Steel, Z., \& Blaszczynski, A. (1998). Impulsivity, personality disorders and pathological gambling severity. Addiction, 93(6), 895-905. https://doi.org/10.1046/j.1360$0443.1998 .93689511 . x$

Toneatto T., Cunningham, J., Hodgins, D., Adams, M., Turner, N., \& Koski-Jannes, A. (2008). Recovery from problem gambling without formal treatment. Addiction Research \& Theory, 16(2), 111-120. https://doi. org/10.1080/16066350801923638

Toneatto, T., Blitz-Miller, T., Calderwood, K., Dragonetti, R., \& Tsanos, A. (1997). Cognitive distortions in heavy gambling. Journal of Gambling Studies volume, 3, 253-266. https:// doi.org/10.1023/A:1024983300428

van Hamel, A., Derwensky, J., Takane, Y., Dlcho , L., \& Gupta, R. (2007). Adolescent Gambling and Coping; within Generalized High-risk Behavior Framework. Journal of Gambling Studies, 23(4), 377-393. http://doi.org/10.1007/s10899007-9066-4 
Vitaro, F., Wanner, B., Carbonneau, R., \& Tremblay, R. (2007). La pratique des jeux de hasard et d'argent, les comportements délinquants et la consommation problématique de substances psychotropes: une perspective développementale. Criminologie, 40(1), 59-77. https://doi. org/10.7202/016015ar
Wieczorek, Ł., \& Dąbrowska, K. (2020). Difficulties in treatment of people with comorbid. Journal of Substance Use, 25(4), 350-356. https://doi.org/10.1080/14659891.2019.17040 78

\section{Pour citer l'article}

Dansereau Touchette, N. (2021). Impact de la comorbidité dans le traitement des joueurs pathologiques. Psycause: Revue scientifique étudiante de l'École de psychologie de l'Université Laval, 11(1), 15-23.

\section{Droits d'auteur}

(C) 2021 Dansereau Touchette. Cet article est distribué en libre accès selon les termes d'une licence Creative Commons Attribution 4.0 International (de type CC-BY 4.0) qui permet l'utilisation du contenu des articles publiés de façon libre, tant que chaque auteur ou autrice du document original à la publication de l'article soit cité(e) et référencé(e) de façon appropriée. 